\title{
Genetic Duplication Process
}

National Cancer Institute

\section{Source}

National Cancer Institute. Genetic Duplication Process. NCI Thesaurus. Code C16607.

Any DNA sequence rearrangement that results in the gain of copies of a genomic region, typically in the form of long interspersed sequences, short interspersed sequences, satellite sequences and retrotransposons. The appearance of duplications of DNA within the genome plays an important role in the diversification of genomes. 